\title{
ESTABILIZAÇÃO DE UM SOLO DA CAMADA CINZA DA FORMAÇÃO GUABIROTUBA PARA FINS DE PAVIMENTAÇÃO URBANA EM CURITIBA, BRASIL
}

\section{STABILIZED SOIL FROM GRAY LAYER OF GUABIROTUBA FORMATION FOR URBAN PAVING PURPOSES IN CURITIBA, BRAZIL}

Jair de Jesús Arrieta Baldovino, Ronaldo Luis dos Santos Izzo

Universidade Tecnológica Federal do Paraná - UTFPR, PR/Brasil.

E-mail: yaderbal@hotmail.com; izzo@utfpr.edu.br

RESUMO - A Formação Guabirotuba está localizada sobre a Bacia Sedimentar da cidade de Curitiba (Brasil). A camada cinza da Formação se estende desde 1 até $50 \mathrm{~m}$ de profundidade. Embora seja a camada mais característica da Formação ainda não se tem estudos de estabilização destes solos para fins de pavimentação urbana na cidade. Assim, este artigo apresenta um estudo experimental da estabilização de um solo siltoso da camada cinza com cimento Portland (CP) empregando tempos de cura $(\mathrm{t})$ de 7,14 e 28 dias. Foram empregados teores de cimento $(\mathrm{C})$ de 3, 5, 7 e $9 \%$ em relação à massa seca do solo. Após os tempos de cura foram realizados ensaios de resistência à compressão simples $\left(q_{\mathrm{u}}\right)$ e de durabilidade empregando ciclos de molhagem/secagem $(\mathrm{M} / \mathrm{S})$. Os resultados demostram um aumento de $\mathrm{q}_{\mathrm{u}}$ com o aumento da quantidade de cimento, com o aumento da densidade de moldagem e com aumento do tempo de cura. Além disso, a durabilidade das misturas aumentou quando adicionado maior quantidade de cimento. Foi verificado que os valores de $q_{u}$ são dependentes da relação semi-empírica porosidade/cimento $\left(\eta / C_{i v}\right)$. Finalmente, foi verificado que $5 \%$ é o teor mínimo de cimento para o uso do solo para fins de pavimentação.

Palavras-chave: Formação Guabirotuba, estabilização, pavimentação, durabilidade.

ABSTRACT - The Guabirotuba Formation is located over the sedimentary basin of the city of Curitiba (Brazil). The gray layer of the Formation extends from 1 to $50 \mathrm{~m}$ deep. Although it is the most characteristic layer of the Formation, there are no studies of stabilization of these soils for urban paving purposes in the city. Thus, this paper presents an experimental study of the stabilization of gray silt soil with Portland cement (PC) using cure times (t) of 7, 14, and 28 days. Cement contents (C) of 3, 5, 7, and $9 \%$ in relation to soil dry mass were used. After cure times, unconfined compressive strength $\left(q_{u}\right)$ and durability tests were performed using wet/dry cycles (W/D). The 
results show an increase of $\mathrm{q}_{\mathrm{u}}$ with increasing cement content, increasing molding density and increasing curing time. In addition, the durability of the mixtures increased when more cement was added. It was found that the values of $q_{u}$ are dependent on the semi-empirical porosity/cement ratio $\left(\eta / C_{i v}\right)$. Finally, $5 \%$ is the minimum cement content for using the soil in paving purposes.

Keywords: Guabirotuba Formation, stabilization, paving, durability.

\section{INTRODUÇÃO}

Uma das técnicas para melhorar as propriedades físico-mecânicas dos solos é através da adição de cimento Portland. $O$ cimento tem sido utilizado para estabilizar solos para uso em camadas de pavimentos, para reforço de solos usados como suporte de fundações superficiais, proteção de encostas e taludes e para construção de fundações profundas. A técnica do solocimento vem sendo empregada há 100 anos (Firoozi et al., 2017).

Recentemente, têm sido realizados vários estudos (e.g., Baldovino et al., 2018a, 2019b; Moreira et al., 2019, 2020) de estabilização química e mecânica em solos da Formação Guabirotuba (FG), localizada sobre a bacia sedimentar de Curitiba, Brasil como mostrado na Figura 1. Os solos da FG se caracterizam por ser expansivos e de baixa capacidade de carga. As camadas mais superficiais se caracterizam por apresentar diferentes cores, indo desde rosa, amarela, vermelha até cinza e marrom. Baldovino et al. (2018a, 2018b, 2019a) estudaram a influência de cal hidratada nas propriedades mecânicas de vários siltes da FG. Os autores estudaram a evolução da resistência à compressão simples e tração por compressão diametral dependentes do índice porosidade/cal $\left(\eta / L_{\text {iv }}\right)$ para curtos tempos de cura (7 dias) e para longos tempos de cura (180 e 360 dias). Todos os solos tiveram melhoras na sua resistência ao longo do tempo.

Moreira et al. (2019) estudaram os efeitos da adição de cimento, resíduo de telha moída, densidade de moldagem e relação porosidade/cimento $\left(n / C_{\text {iv }}\right)$ no $q_{u}$ de um solo siltoso. Os autores calcularam que todas as resistências são dependentes de $\eta / C_{i v}$ e que a telha moída não é um material que melhore a capacidade de carga das misturas. Imediatamente, Moreira et al. (2020) estabilizaram mecanicamente um solo siltoso com resíduos da construção civil para aplicação em pavimentação. Foram obtidos resistências e módulos resilientes satisfatórios para empregar as misturas como sub-base de pavimento.

Finalmente, Baldovino et al. (2019b, 2020a, 2020b) e Baldovino e Izzo (2019) usaram o cimento como estabilizante de vários solos siltosos da FG. Os autores concluíram que $\circ \mathrm{q}_{\mathrm{u}}$ e $\mathrm{q}_{\mathrm{t}}$ de todos os solos da FG são dependentes de $\eta / C_{i v}$ e dos tempos de cura. Adicionalmente, se tem encontrado que $\mathrm{q}_{\mathrm{t}}$ é aproximadamente $16 \%$ do valor de $q_{u}$ (i.e. $q_{t}=0,16 q_{u}$ ).

A pesar da existência de várias pesquisas sobre a estabilização dos solos da FG com cimento, cal e resíduos, ainda não se tem estudos de melhoramento mecânico dos solos da camada de cor cinza que chega até $50 \mathrm{~m}$ de profundidade. Assim, este estudo analisa os efeitos de adição de cimento sobre as propriedades mecânicas e de durabilidade de um silte da camada cinza da FG para fins de pavimentação urbana na cidade de Curitiba, Brasil. 
Figura 1. Localização da Formação Guabirotuba (FG). Modificado do Mapa Geológico do Estado do Paraná. Instituto de Terras, Cartografia e Geologia do Paraná - ITCG (MINEROPAR, 2020).

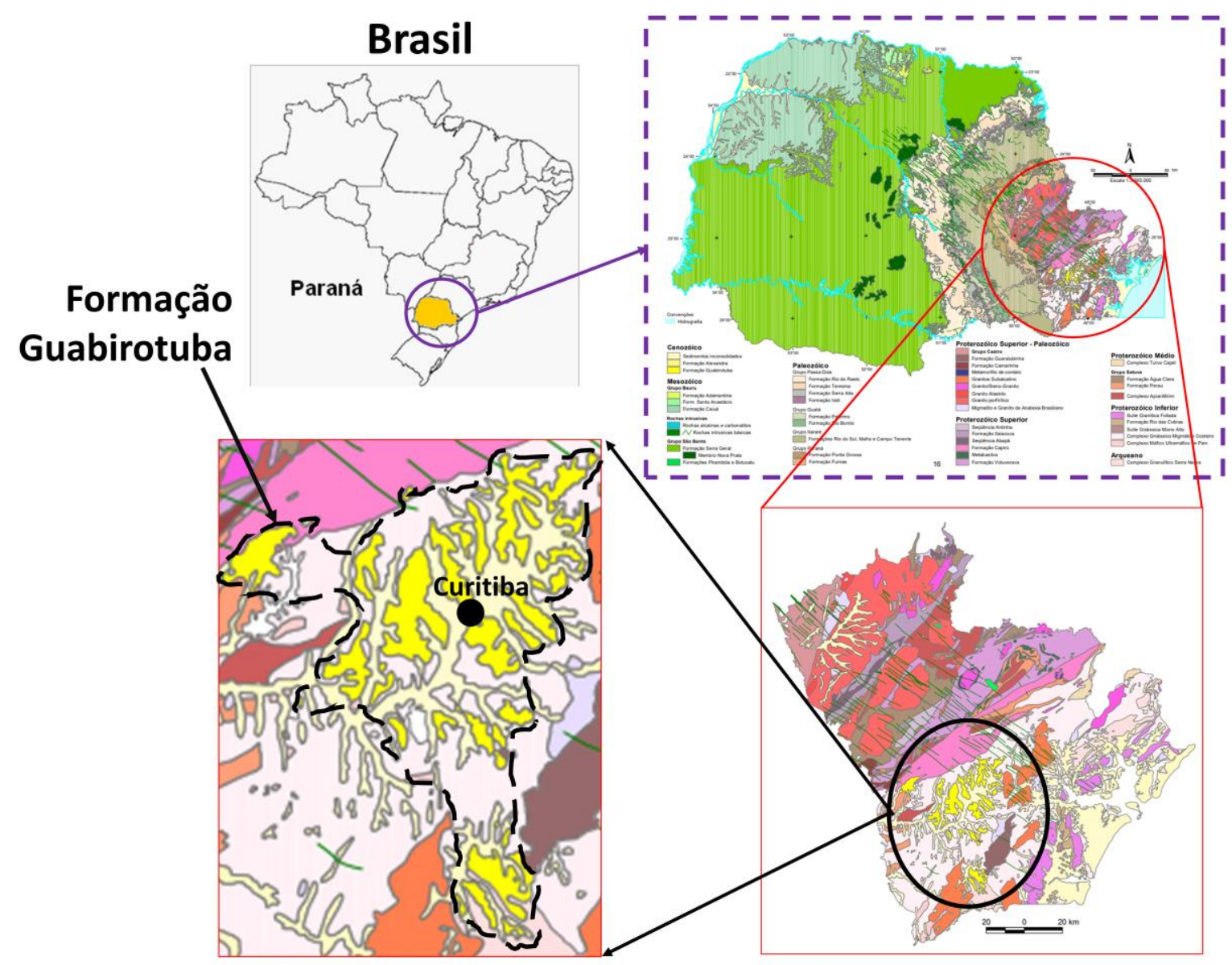

Fonte: Os autores.

\section{PROGRAMA EXPERIMENTAL}

O programa experimental foi dividido em três etapas: a primeira foi a realização dos ensaios de caracterização do solo de cor cinza e do cimento: granulometria do solo de acordo à norma americana ASTM D2487 (ASTM 2011), limites de Atterberg do solo de acordo às normas brasileiras NBR 7180 (ABNT 2016a) e NBR 6459 (ABNT 2016b), a massa especifica real dos grãos do solo de acordo à norma ASTM D854 (ASTM 2014), massa especifica real dos grãos do cimento de acordo à norma brasileira NBR 16605 (ABNT 2017) e as propriedades de compactação do solo nas duas energias tradicionais (normal e modificada) seguindo norma brasileira NBR 7182 (ABNT 2016c); a segunda etapa, consistiu-se na moldagem, cura e rompimento dos corpos de prova solocimento submetidos a ensaios de compressão simples. A terceira e última etapa consistiu-se na moldagem, cura e realização dos ensaios de durabilidade por ciclos de molhagem/secagem (M/S) medindo a perda de massa acumulada (Pma).

\subsection{Materiais}

No presente trabalho foram utilizados três materiais: solo, cimento Portland de alta resistência inicial (CP V-ARI) e água destilada. A amostra de solo foi coletada na zona leste da cidade de Curitiba (Brasil) no município de São José dos Pinhais de maneira manual em estado deformado, evitando uma possível contaminação e em quantidade suficiente para a realização de todos os ensaios.

O solo foi coletado em um talude rodoviário a 1,5-2,0 $\mathrm{m}$ de profundidade. Os diâmetros das partículas de solo correspondentes aos 10\%, 30\%, 50\%, 60\% e 
90\% de material passante foram calculados como $D_{10}=0,01 \mathrm{~mm}, D_{30}=0,024 \mathrm{~mm}, D_{50}=0,04$ $\mathrm{mm}, D_{60}=0,055 \mathrm{~mm}$ e $D_{90}=0,28 \mathrm{~mm}$, respectivamente. Adicionalmente, $\mathrm{O}$ coeficiente de uniformidade (i.e. $C_{\mathrm{u}}=D_{60} / D_{10}$ ) e o coeficiente de curvatura [i.e. $\left.C_{c}=\left(D_{30}\right)^{2} /\left(D_{10} / D_{60}\right)\right]$ foram calculados como $C_{u}=5,5$ e $C_{c}=1,05$, respectivamente. Assim, segundo o Sistema Unificado de Classificação de Solos (ASTM, 2011), o solo é classificado como um silte arenoso de baixa compressibilidade (ML). O solo apresentou uma pequena variação nos percentuais de cada tipo de componente, na qual a porcentagem de areia média alcançou 15\%; a areia fina chegou em $25 \%$ e para o silte se obteve $60 \%$, sendo a porcentagem de silte, compreendia na fração de 0,002 $\mathrm{mm}<$ diâmetro $<0,06 \mathrm{~mm}$ compõe a maior parcela do solo. Os resultados dos ensaios de caracterização física do solo são apresentados na Tabela 1.
O cimento foi fornecido por um fabricante local. A Tabela 2 apresenta as propriedades físico-químicas do cimento. As propriedades químicas foram fornecidas pelo fabricante e as físicas foram calculadas no laboratório. De acordo com a Tabela 2 o cimento CP $\vee$ tem uma densidade de 3,11 $\mathrm{g} / \mathrm{cm}^{3}$.

Para a realização de todos os ensaios de caracterização do solo, das misturas solocimento e para a moldagem de corpos de prova foi usada água destilada a $24 \pm 3^{\circ} \mathrm{C}$ para evitar reações não desejadas e limitar o número de variáveis no estudo.

Tabela 1. Propriedades físicas do solo

\begin{tabular}{|l|c|c|}
\hline \multicolumn{1}{|c|}{ Propriedade } & Valor & Norma seguida \\
\hline Limite de liquides, $\%$ & 44,74 & (ABNT, 2016a) \\
\hline Limite de plasticidade, $\%$ & 26,43 & (ABNT, 2016b) \\
\hline Índice de plasticidade, $\%$ & 18,31 & - \\
\hline Massa especifica real dos grãos $\left(\mathrm{G}_{\mathrm{s}}\right)$ & 2,83 & (ASTM, 2014) \\
\hline Areia meia $(0,2 \mathrm{~mm}<$ diâmetro $<0,6 \mathrm{~mm}), \%$ & 15 & (ABNT, 1995) \\
\hline Areia fina $(0,06 \mathrm{~mm}<$ diâmetro $<0,2 \mathrm{~mm}), \%$ & 25 & (ABNT, 1995) \\
\hline Silte $(0,002 \mathrm{~mm}<$ diâmetro $<0,06 \mathrm{~mm}), \%$ & 60 & (ABNT, 1995) \\
\hline Argila (diâmetro $<0,002 \mathrm{~mm}), \%$ & 0 & (ABNT, 1995) \\
\hline Diâmetro efetivo $\left(D_{10}\right), \mathrm{mm}$ & 0,01 & - \\
\hline Diâmetro médio das partículas $\left(D_{50}\right), \mathrm{mm}$ & 0,04 & - \\
\hline Coeficiente de uniformidade $\left(C_{\mathrm{u}}\right)$ & 5,5 & - \\
\hline Coeficiente de curvatura $\left(C_{\mathrm{c}}\right)$ & 1,05 & - \\
\hline Classificação do solo $(S U C S)$ & $\mathrm{ML}$-Silte arenoso & (ASTM, 2011) \\
\hline Cor & Cinza & - \\
\hline pH em água & 4,7 & - \\
\hline Teor de umidade ótimo (Proctor normal), \% & 27,0 & (ABNT, 2016c) \\
\hline Peso específico seco máximo (do Proctor normal), $\mathrm{kN} / \mathrm{m}^{3}$ & 14,52 & (ABNT, 2016c) \\
\hline Teor de umidade ótimo (energia modificada), $\%$ & 17,6 & (ABNT, 2016c) \\
\hline Peso específico seco máximo (energia modificada), $\mathrm{kN} / \mathrm{m}^{3}$ & 17,57 & (ABNT, 2016c) \\
\hline
\end{tabular}

Fonte: Os autores 
Tabela 2. Propriedades físico-químicas do cimento Portland CP V

\begin{tabular}{l|r}
\multicolumn{1}{c|}{ Propriedade } & \multicolumn{1}{|c}{ Valor } \\
\hline$\% \mathrm{MgO}$ & 4,11 \\
\hline$\% \mathrm{SO}_{3}$ & 2,99 \\
\hline$\% \mathrm{CaO}$ & 60,73 \\
\hline$\% \mathrm{Al}_{2} \mathrm{O}_{3}$ & 4,38 \\
\hline$\% \mathrm{Fe}_{2} \mathrm{O}_{3}$ & 2,83 \\
\hline$\% \mathrm{SiO}_{2}$ & 19,9 \\
\hline$\%$ Resíduo insolúvel & 0,77 \\
\hline $\mathrm{q}_{\mathrm{u}}$ aos 7 dias $(\mathrm{MPa})$ & 42 \\
\hline $\mathrm{qu}_{\mathrm{u}}$ aos 28 dias $(\mathrm{MPa})$ & 53 \\
\hline$\%$ Finura & 0,04 \\
\hline Densidade $\gamma_{\mathrm{c}}-\mathrm{g} / \mathrm{cm}^{3}$ & 3,11 \\
\hline
\end{tabular}

Fonte: Os autores.

\subsection{Definição dos pontos de moldagem e tempos de cura}

Os pontos de moldagem foram estabelecidos após a realização dos ensaios de compactação do solo nas duas energias: normal e modificada, de acordo com a norma brasileira NBR 7182 (ABNT 2016c). A Tabela 3 mostra os pontos de moldagem propostos para compactar as misturas solo-cimento.

Tabela 3. Pontos de moldagem

\begin{tabular}{l|l|l|l|l|l|l}
\hline \multirow{2}{*}{$\begin{array}{c}\text { Cimento } \\
(\%)\end{array}$} & \multicolumn{2}{|l|}{$\begin{array}{l}\text { Peso específico } \\
\text { seco }\left(\mathbf{k N} / \mathbf{m}^{3}\right)\end{array}$} & \multicolumn{2}{l}{$\begin{array}{l}\text { Teor de umidade } \\
\text { de moldagem (\%) }\end{array}$} \\
\cline { 2 - 8 } & A & B & C & A & B & C \\
\hline 3 & 14 & 15 & 16 & 23 & 23 & 23 \\
\hline 5 & 14 & 15 & 16 & 23 & 23 & 23 \\
\hline 7 & 14 & 15 & 16 & 23 & 23 & 23 \\
\hline 9 & 14 & 15 & 16 & 23 & 23 & 23 \\
\hline
\end{tabular}

Fonte: Os autores.

Com o objetivo de estudar a influência da massa específica seca e dos vazios sobre a resistência mecânica do solo cimentado artificialmente com o cimento mencionado, foram definidos 3 pontos de moldagem: A $\left(14 \mathrm{kN} / \mathrm{m}^{3}\right), B\left(15 \mathrm{kN} / \mathrm{m}^{3}\right)$ e C $\left(16 \mathrm{kN} / \mathrm{m}^{3}\right)$. Estes pontos de moldagem foram definidos estrategicamente considerando possíveis condições de campo. Pontos de moldagem estratégicos para estudar solos melhorados já foram utilizados anteriormente por Rios et al. (2012), Baldovino et al. (2018a), Baldovino et al. (2018b), Baldovino et al. (2020a) e Moreira et al. (2019). Todos os corpos de prova de solocimento foram submetidos a ensaios depois de 7, 14 e 28 dias de cura em condições de saturação para anular o máximo possível a influência da sucção sobre a resistência nas misturas. Para cada ponto de moldagem foram confeccionados 36 corpos de prova (12 para cada tempo de cura).

\subsection{Preparação dos corpos de prova}

Para os ensaios de compressão simples foram moldados 108 corpos de prova de $100 \mathrm{~mm}$ de altura e $50 \mathrm{~mm}$ de diâmetro (volume de $196,4 \mathrm{~cm}^{3}$ ). Para os ensaios de durabilidade foram moldados em total 4 corpos de prova de $10 \mathrm{~cm}$ de diâmetro e $12,73 \mathrm{~cm}$ de altura (volume de $1000 \mathrm{~cm}^{3}$ ).

Depois da coleta em campo, o solo foi seco em estufa à temperatura de $100 \pm 5^{\circ} \mathrm{C}$, e colocado em porções uniformemente distribuídas para ser misturado com o cimento. Adicionou-se a quantidade de cimento seco com referência ao peso seco da amostra de solo em quatro teores diferentes de adição (3, 5, 7 e 9\%) de acordo a recentes estudos sobre estabilização química de solos da Formação Guabirotuba (Baldovino et al. 2019a; Moreira et al. 2019). Realizou-se a mistura do solo com o cimento de forma manual de modo que a mistura ficasse a mais homogênea possível. Em seguida, foi adicionada uma porcentagem de água em massa, sendo esta porcentagem referente ao teor de umidade dos pontos de moldagem estabelecidos na Tabela 3. A mistura do solo-cimento com a água destilada foi realizada em um período não superior a 7 minutos, com isto tentando minimizar as reações do cimento com a água antes do processo de moldagem dos corpos de prova. As amostras para a moldagem dos corpos de prova foram compactadas estaticamente em três camadas com um molde de aço inox. Para assegurar a massa específica seca, foi realizado o cálculo do volume do molde e da massa da mistura úmida necessários para cada corpo de prova. Após esses cálculos, foi pesada a quantidade necessária de material para cada corpo de prova. Foram necessárias 3 camadas para compactar os corpos de prova, sendo a 
primeira e a segunda camada escarificadas para maior homogeneidade. A moldagem foi feita com a ajuda de uma prensa hidráulica manual com capacidade de 10 ton. Depois de cada processo de moldagem, três amostras da mistura foram utilizadas para medir o teor de umidade em estufa durante 24 horas a $100 \pm 5^{\circ} \mathrm{C}$.

Os corpos de prova foram pesados em uma balança de precisão de 0,01 g e as dimensões dele eram medidas com o uso de um paquímetro de 0,1 $\mathrm{mm}$ de precisão. Os corpos de provas extraídos foram envolvidos com plástico filme transparente para manter o teor de umidade. Por último, os corpos de prova de compressão simples foram armazenados em câmara úmida para processo de cura durante 7,14 e 28 dias (a temperatura média de $24^{\circ} \pm 3^{\circ} \mathrm{C}$ ) para prevenir mudanças significativas de umidade até o dia do ensaio. Os corpos de prova para ensaios de durabilidade foram curados durante 7 dias.

As amostras tinham que respeitar as seguintes tolerâncias máximas para serem usadas nos ensaios de compressão simples e durabilidade: dimensões das amostras com diâmetro de $\pm 0,5 \mathrm{~mm}$ e altura de $\pm 1 \mathrm{~mm}$, peso específico aparente seco $\left(\gamma_{\mathrm{d}}\right)$ de $\pm 1 \%$ e teor de umidade $(\omega)$ de $\pm 0,5 \%$. Para cada ponto de moldagem e teor de cimento foram moldados 3 corpos de prova. Para ensaios de compressão simples, após os 7, 14 e 28 dias de cura, os corpos de prova eram imersos em um tanque com água destilada durante 6 horas antes do ensaio para garantir ao máximo sua saturação e evitar com isto a influência da sucção na resistência. Depois da imersão, eram secados superficialmente com um pano seco.

\subsection{Ensaios de resistência à compressão} simples e durabilidade

Para a realização dos ensaios de compressão simples foi usada uma prensa automática com célula de carga com capacidade de $30 \mathrm{kN}$. Os ensaios foram feitos com um sistema automatizado, medindo, principalmente, a força aplicada, com resolução de $2,5 \mathrm{~N}$, a deformação, com sensibilidade de 0,01 $\mathrm{mm}$, sendo a velocidade do ensaio de $1,5 \mathrm{~mm} / \mathrm{min}$. Os procedimentos dos ensaios de compressão simples seguiram a norma brasileira NBR 5739 (ABNT 2018). A Figura 2 apresenta um corpo de prova solo-cimento ensaiado à compressão.

Os ensaios de durabilidade com ciclos de molhagem/secagem seguiram as indicações da norma americana ASTM D55915 (ASTM, 2015). Após a cura dos corpos de prova por 7 dias, estes foram colocados em um tanque com água para imersão durante 5h. Logo do processo de imersão, foram levados à estufa a $70^{\circ} \mathrm{C}$ durante $42 \mathrm{~h}$. Posteriormente os corpos de prova foram retirados do processo de secagem e foram escovados. Foram necessárias de 18 a 20 escovadas cobrindo a área lateral dos corpos de prova 2 vezes, e 4 escovadas cobrindo as áreas transversais. Foi anotada a massa perdida pelo corpo de prova após cada ciclo até completar um total de 12 ciclos.

Figura 2. Corpo de prova solo-cimento após ensaio de compressão simples

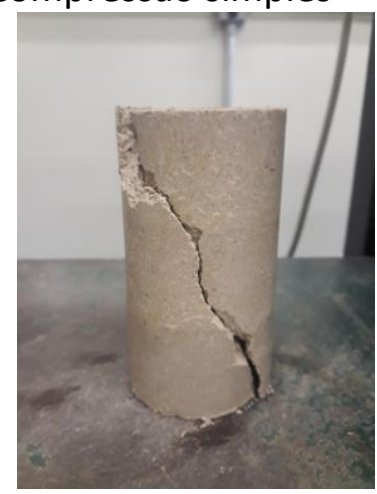

Fonte: Os autores.

\section{APRESENTAÇÃO, ANÁLISE E DISCUSSÃO DOS RESULTADOS}

3.1. Ensaios de resistência à compressão simples

É apresentado na Figura 3 os efeitos da adição do cimento e do tempo de cura (t) na resistência à compressão simples do solo estabilizado em planos 3D. Cada plano mostrado na Figura 3 representa um ponto de moldagem (Tabela 3), sendo que o plano menos inclinado representa a resistência $q_{u}$ 
dos corpos de prova moldados no peso especifico seco de $14 \mathrm{kN} / \mathrm{m}^{3}$ e o plano com maior inclinação representa os CPs moldados em $16 \mathrm{kN} / \mathrm{m}^{3}$. Assim, os planos são controlados por uma única equação do tipo exponencial para cada peso específico usado na moldagem. As Equações (1)-(3) representam o crescimento dos valores de $\mathrm{q}_{\mathrm{u}}$ para $14 \mathrm{kN} / \mathrm{m}^{3}$ (i.e. porosidade $\eta=50,7 \%$ ), 15 $\mathrm{kN} / \mathrm{m}^{3}$ (i.e. $\eta=47 \%$ ) e $16 \mathrm{kN} / \mathrm{m}^{3}$ (i.e. $\eta=43,7 \%$ ), respectivamente, onde t é o tempo de cura.

$q_{u}=2272,7 \times e^{-0,5\left(\left(\frac{\mathrm{t}-28,9}{22,6}\right)^{2}+\left(\frac{\mathrm{C}-11,4}{5,3}\right)^{2}\right)}$

$$
q_{u}=3848,4 \times e^{-0,5\left(\left(\frac{\mathrm{t}-68,7}{54,7}\right)^{2}+\left(\frac{\mathrm{C}-9,6}{4,4}\right)^{2}\right)}
$$

$q_{u}=5178,0 \times e^{-0,5\left(\left(\frac{\mathrm{t}-36,1}{30,4}\right)^{2}+\left(\frac{\mathrm{C}-10,0}{4,5}\right)^{2}\right)}$

É mostrado na Figura 3 que, $\mathrm{q}_{\mathrm{u}}$ aumenta quando é aumentado o teor de cimento e o tempo de cura. $\mathrm{O}$ cimento desenvolve fortes ligações entre as partículas minerais do solo, resultando em uma matriz cimentada, que envolve os grãos. A estrutura alveolar da matriz é responsável pela resistência do solocimento. A adesão entre as partículas de argila dentro da matriz é bastante baixa. As ligações impedem que as partículas se movam uma em direção à outra, minimizando a plasticidade e aumentando a resistência ao cisalhamento. As partículas de argila são coaguladas pela cal liberada durante a hidratação do cimento, reduzindo sua afinidade pela água e, portanto, diminuindo propriedades de inchamento $e$ encolhimento do solo (Baldovino et al. 2020b).

As Eqs. (1)-(3) apresentam uma mesma forma que é descrita pela Eq. (4).

$q_{u}=\mathrm{S} \times e^{-k}$

Onde $S$ é constante, nas mesmas unidades de $\mathrm{q}_{\mathrm{u}}(\mathrm{kPa})$ e $\boldsymbol{k}$ é dependente de $\mathrm{t}$ e $\mathrm{C}$. Notase na Figura 3 que $S$ está fortemente ligada com o aumento da densidade de moldagem. Os maiores valores de $q_{u}$ foram obtidos quando adicionado $9 \% \mathrm{C}$. Foram obtidas resistências máximas de 3000, 4000 e 5000 kPa (adicionado 9\%C) para 7, 28 e 90-dias de cura. 
Figura 3. Efeitos da adição de cimento e do tempo de cura na resistência à compressão simples das misturas solo-cimento em planos 3D dependendo do peso específico seco de moldagem $\left(\gamma_{\mathrm{d}}\right)$

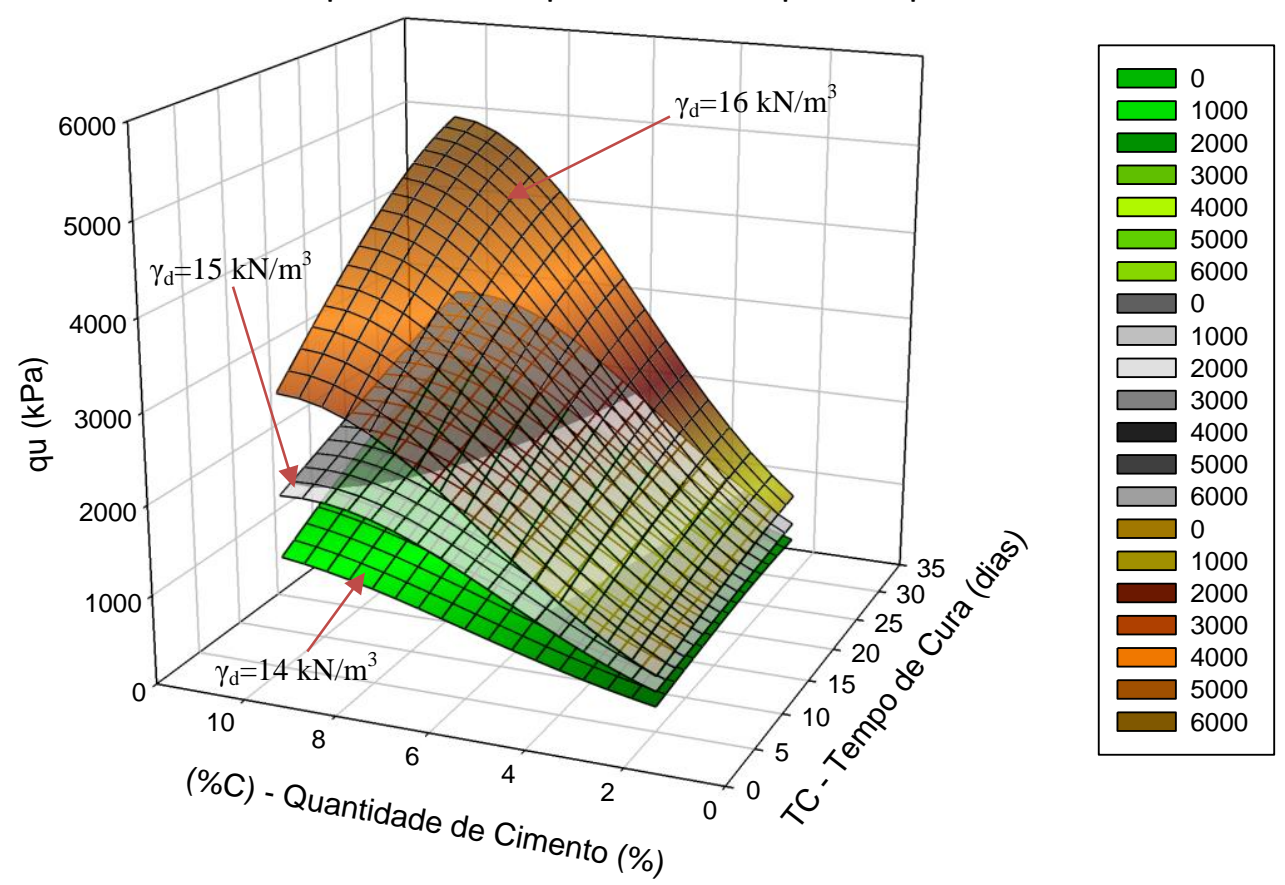

Fonte: Os autores.

Os resultados de $\mathrm{q}_{\mathrm{u}}$ também podem ser apresentados em função do índice porosidade/teor volumétrico de cimento $\left(\eta / C_{i v}\right)$ introduzido na literatura por Consoli et al. (2007). Assim, a influência de $\eta / C_{i v}$ sobre $\mathrm{q}_{\mathrm{u}}$ é apresentada pela Figura 4. $\mathrm{C}_{i v}$ é calculado para cada corpo de prova empregando a Equação (5).

$$
\mathrm{C}_{i v}=\left(\frac{\gamma_{\mathrm{d}}}{1+\mathrm{C} / 100}\left(\frac{\mathrm{C}}{100}\right)\right) / \gamma_{\mathrm{C}}
$$

Onde $\gamma_{d}$ é o peso específico seco aparente da mistura, $\mathrm{C}$ é o teor de cimento e $\gamma_{C}$ é a densidade dos grãos do cimento igual a $3,11 \mathrm{~g} / \mathrm{cm}^{3}$. O teor volumétrico $C_{i v}$ aumenta com o acréscimo do teor de cimento, enquanto a relação porosidade/teor volumétrico do cimento diminui. Para as misturas, a relação $\eta / C_{i v}$ varia de $28-46 \%$. Em média, a faixa de variação de $\eta / C_{i v}$ para cada teor de cimento é de 3,$6 ; 4,5 ; 6,3$ e 10,5 para $9,7,5$ e $3 \%$, respectivamente. Ou seja, a faixa de variação cresce com a diminuição da quantidade de cimento no corpo de prova, e, quando a faixa de variação diminui fornece os maiores valores de resistência à compressão simples. Pode-se observar na Figura 4 que existe uma tendência potencial de $q_{u}$ dependente de $\eta / C_{i v}$ para todos os teores de cimento. Para fazer compatível a variação de $C_{i v}$ e $q_{u}$, foi necessário ajustar os valores de $C_{i v}$ a 0,25 . O expoente de 0,25 sobre o teor volumétrico de cimento $\left(C_{i v}\right)$ forneceu altos coeficientes de determinação maiores a $0,97\left(R^{2}>0,97\right)$. 
Figura 4. Efeitos da relação porosidade/teor volumétrico de cimento sobre a resistência à compressão simples das misturas solo-cimento dependendo do tempo de cura

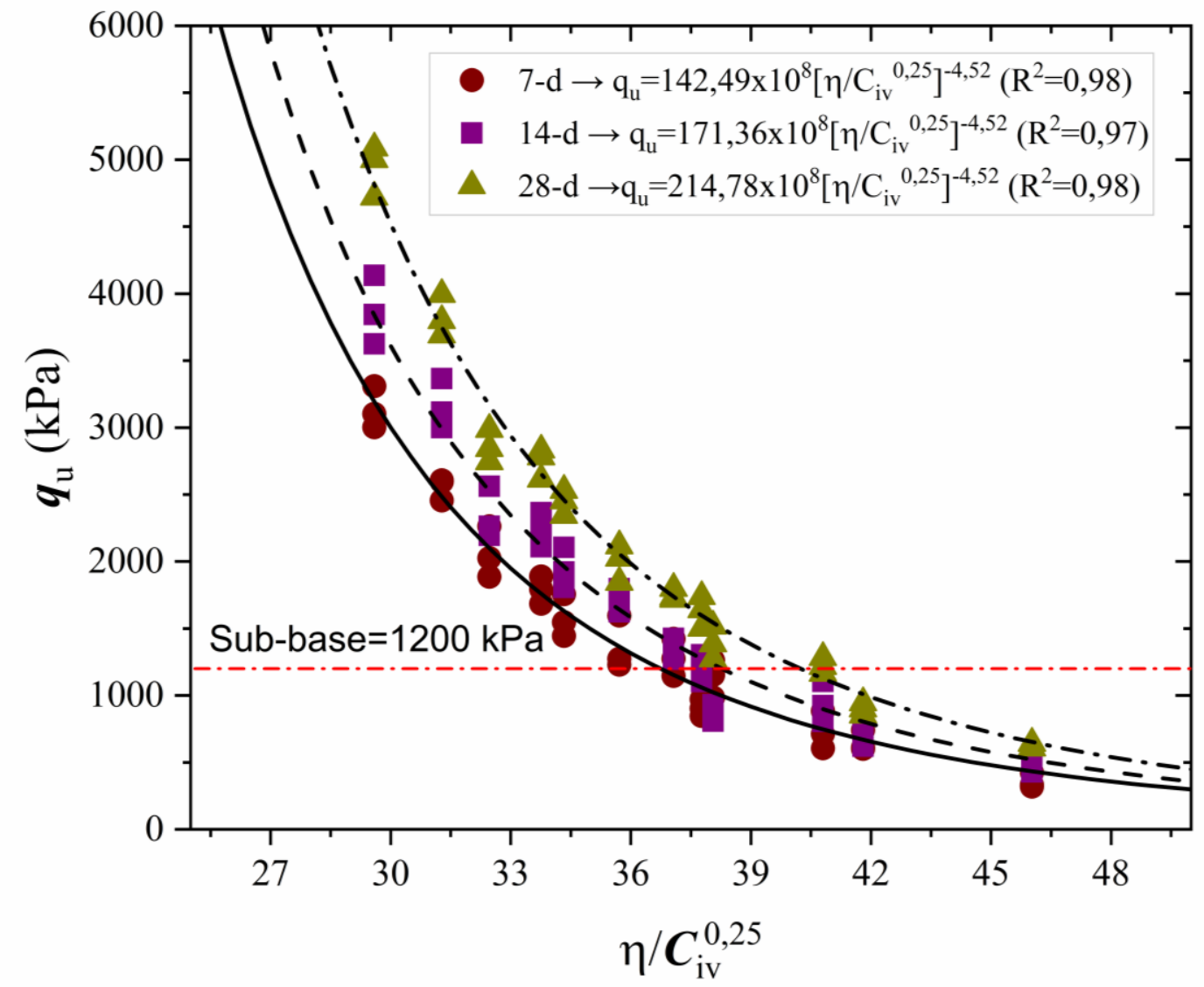

Fonte: Os autores.

As Equações 6-8 definem o crescimento de $q_{u}$ com a diminuição de $\eta / C_{i v}{ }^{0,25}$ para 7,14 e 28 dias de cura respectivamente:

$$
\begin{aligned}
& \mid q_{u}=142,49 \times 10^{8}\left[\frac{\eta}{\left(C_{i v}\right)^{0,25}}\right]^{-4,52}\left(R^{2}=0,98\right) \\
& \mid q_{u}=171,36 \times 10^{8}\left[\frac{\eta}{\left(C_{i v}\right)^{0,25}}\right]^{-4,52}\left(R^{2}=0,97\right) \\
& \mid q_{u}=214,78 \times 10^{8}\left[\frac{\eta}{\left(C_{i v}\right)^{0,25}}\right]^{-4,52}\left(R^{2}=0,98\right)
\end{aligned}
$$

Pode-se observar que as Equações 6-8 seguem a forma: $q_{u}=A\left[\frac{\eta}{c_{i v}{ }^{C}}\right]^{-B}$. Baseado nos estudos feitos por Consoli et al. (2013) e Baldovino (2018d), os valores de B e C depende do tipo de solo. Segundo Diambra et al. (2017) existe uma correlação entre B e C para areias cimentadas, onde $C=1 / B$. No presente estudo usando um silte cimentado, nota-se que o valor de $C=0,50 / B$. A relação $\eta / C_{i v}{ }^{C}$ é capaz de fornecer uma tendência única da resistência $q_{u}$ do solo siltoso experimentado no presente trabalho cimentado artificialmente com um cimento de alta resistência inicial (ARI). Para Rios et al. (2012) e Mola-Abasi e Shooshpasha (2016) a relação $\eta / C_{i v}$ demonstra ser um ótimo parâmetro de ajuste para descrever o comportamento à compressão não confinada de solos estabilizados com cimento.

No Brasil, o Departamento Nacional de Infraestrutura de Transportes (DNIT) em seu manual 143 de 2010 (DNIT 143/2010) estabelece que as misturas de solo-cimento podem ser empregadas em como sub-base de pavimentos quando proporcionem resistências à compressão simples maiores a $1200 \mathrm{kPa}$ aos 7 dias de cura. Na Figura 4 foi plotado o requerimento do DNIT junto às resistências $q_{u}$ de todas as misturas. Foi 
verificado que $5 \%$ de cimento proporciona valores $\mathrm{q}_{\mathrm{u}}$ de $1200 \mathrm{kPa}$. Baldovino et al. (2020) indica que 5\% de cimento é um teor moderado para uso em pavimentação.

\subsection{Equação de estimativa de $q_{u}$}

Se as Equações (6) - (8) são divididas pela expressão $10^{8}\left(\eta / C_{\mathrm{iv}}{ }^{0,25}\right)^{-4,52}$ se obtém uma constante que aumenta com o tempo de cura. A evolução da resistência à compressão simples desde 7 até 28 dias de cura é apresentada na Figura 5. O aumento do tempo de cura produz um aumento nos valores de $q_{\mathrm{u}}$ mostrando um aumento potencial com valor de coeficiente de determinação de $R^{2}=0,99$. A equação de dosagem para a compressão simples é proposta com a relação $\eta / C_{\text {iv }}$ e com o tempo de cura (t). A expressão de dosagem para $q_{\mathrm{u}}$ é mostrada na Equação (9).

$q_{u}=79,55 t^{0,30} \times 10^{8}\left[\frac{\eta}{\left(C_{i v}\right)^{0,25}}\right]^{-4,52}\left(R^{2}=0,99\right)$

Se cada valor de porosidade e quantidade de cimento em volume é inserido na Equação (9), se obtém a curva de previsão de $q_{u}$ frente aos valores experimentais. Desta maneira, a curva é exibida na Figura 6.

Figura 5. Efeitos do tempo de cura na resistência mecânica normalizada

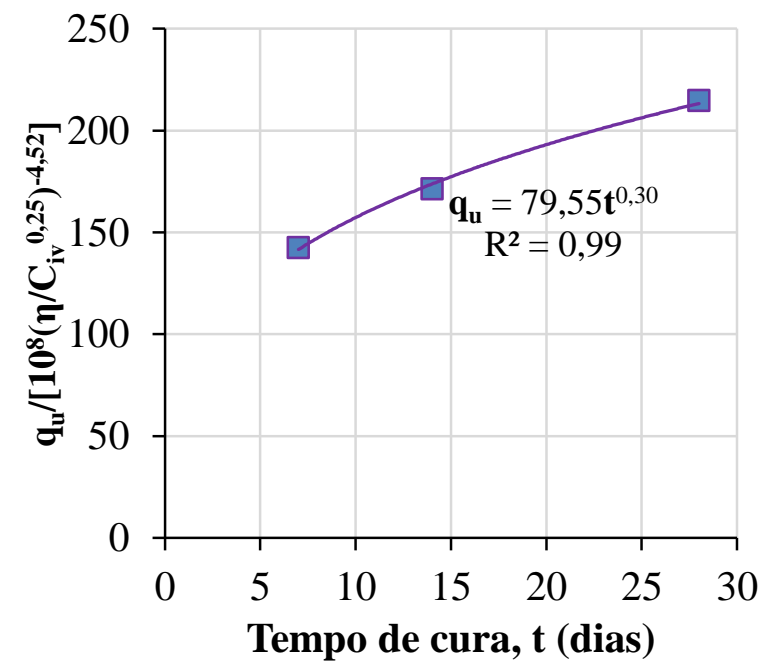

Fonte: Os autores.
Figura 6. Validação da equação de estimativa de $q_{u}$ (valores experimentais vs calculados)

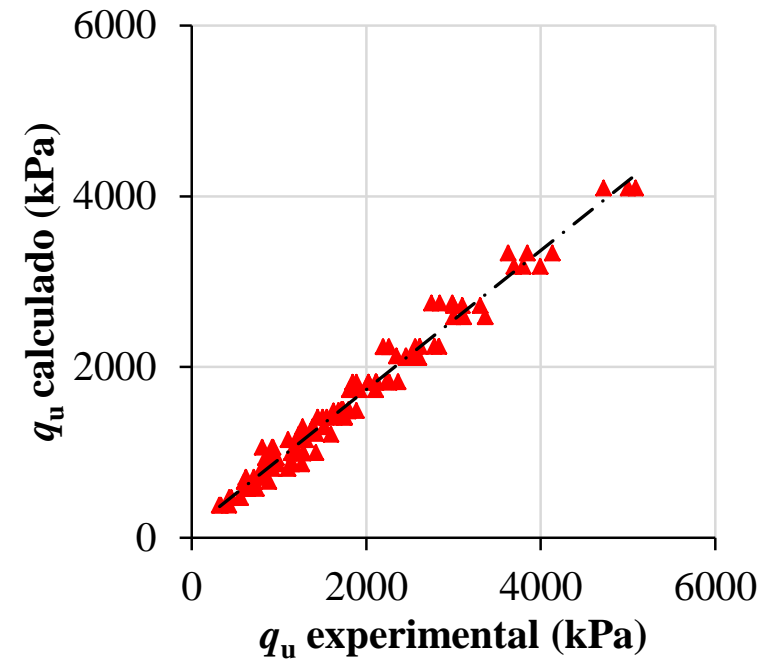

Fonte: Os autores.

\subsection{Ensaios de durabilidade usando ciclos de molhagem/secagem}

Cada ciclo de M/S começou com os corpos de prova imersos em água destilada por 5 horas, secado em estufa por 42 horas e posterior repouso por 1 hora antes da escovação. A aparência de um corpo de prova com 3\% de cimento e mostrado na Figura 7. Adicionalmente, a aparência corpos de prova com teores de $5 \%$, e $9 \%$ de cimento antes do $1 .^{\circ}$ ciclo é apresentado na Figura 8, assim como, também, a aparência depois de 12 ciclos de $\mathrm{M} / \mathrm{S}$.

Observa-se que todos os corpos de prova mantiveram a sua forma cilíndrica, exceto os compactados com $3 \%$ de cimento, os quais foram se deteriorando e perdendo, progressivamente, grandes porcentagens de massa, a amostra com 3\%C moldado em $\gamma_{\mathrm{d}}=14$ $\mathrm{kN} / \mathrm{m}^{3}$ perdeu $100 \%$ da massa no quarto ciclo $\mathrm{M} / \mathrm{S}$. Na Figura 9 pode ser observada a perda de massa acumulada (Pma) das misturas influenciada pelo número de ciclos de $\mathrm{M} / \mathrm{S}$. Observa-se que as misturas com $5 \%$ de cimento tem perdas de massa de $4 \%$ e $9,7 \%$ compactadas em $\gamma_{\mathrm{d}}=14 \mathrm{kN} / \mathrm{m}^{3}$ e $15 \mathrm{kN} / \mathrm{m}^{3}$, respectivamente. A mistura com $9 \%$ de cimento perdeu $0,66 \%$ da sua massa.

A Pma está diretamente relacionada à quantidade de cimento utilizada e vazios iniciais do corpo de prova (Donrak et al., 2020). Por isso, a Pma foi correlacionada à relação $\eta / C_{\text {iv }}$, 
como pode ser visto na Figura 10. O índice vazios/cimento também controla a Pma das misturas compactadas que se eleva com o a umento de $\eta / C_{i v}$. As correlações entre a Pma e índice $\eta / C_{\text {iv }}$ obtiveram excelentes ajustes, com $R^{2} \geq 0,99$.

Figura 7. Corpos de prova com $3 \%$ cimento após o primeiro ciclo de molhagem/secagem

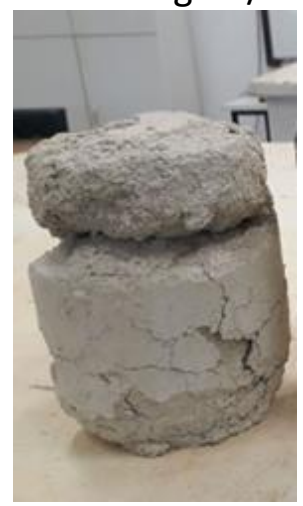

Fonte: Os autores.
Para solos siltosos estabilizados quimicamente para aplicação em pavimentação, o valor de Pma não deve exceder 7-8\%, segundo a PCA (1992). Somente as misturas com $5 \% \mathrm{C}$ e compactadas acima de $15 \mathrm{kN} / \mathrm{m}^{3}$ cumprem com esse requerimento. Assim, $5 \%$ é considerado o teor de cimento mais apropriado para estabilizar o silte cinza da Formação Guabirotuba, baseado nas propriedades mecânicas (Figura 4) e de durabilidade desse tipo de solo.

Figura 8. Corpos de prova para ensaios de durabilidade de solo-cimento. (a) Antes os 12 ciclos M/S. (b) Após os 12 ciclos M/S

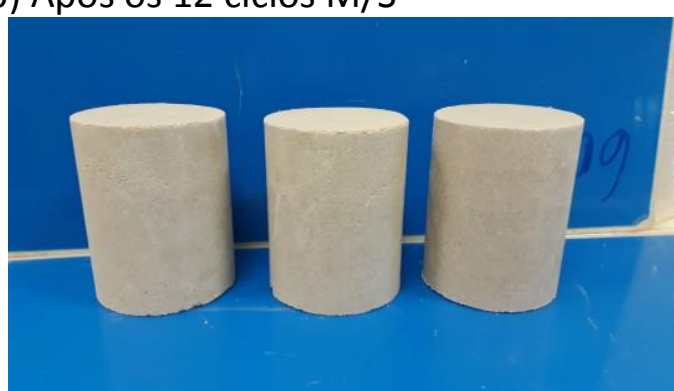

(a) Antes dos ciclos de $\mathrm{M} / \mathrm{S}$ Fonte: Os autores.

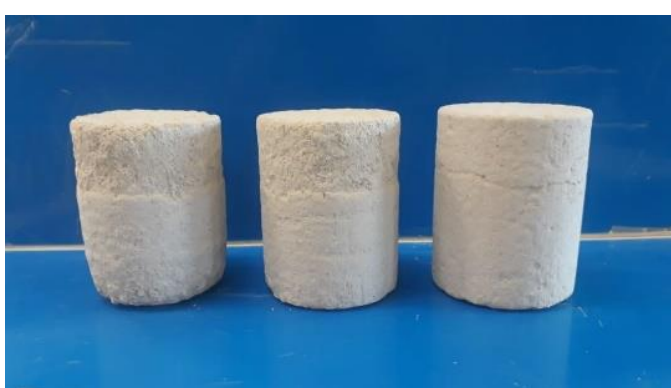

(b) Depois dos ciclos de M/S

Figura 9. Efeitos do ciclo de molhagem/secagem sobre a perda de massa acumulada (Pma) de várias misturas de solocimento.

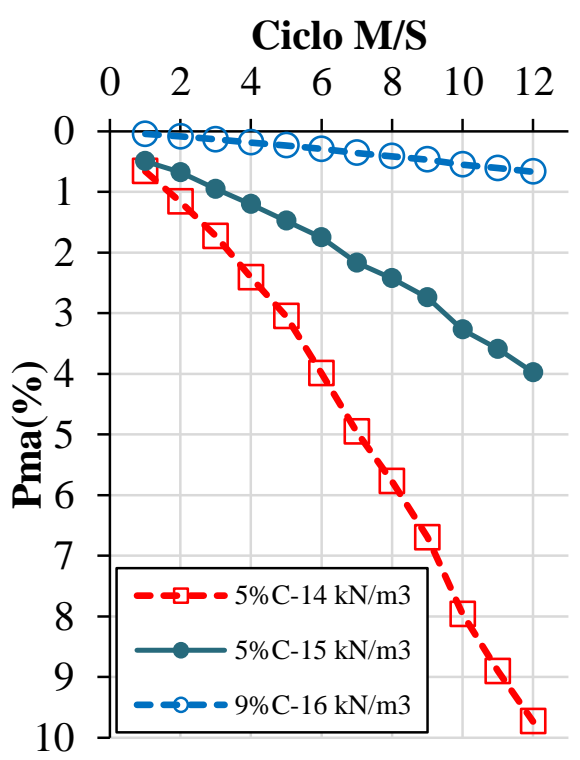

Fonte: Os autores. 
Figura 10. Efeitos da relação porosidade/teor volumétrico de cimento sobre a Pma de várias misturas de solo-cimento.

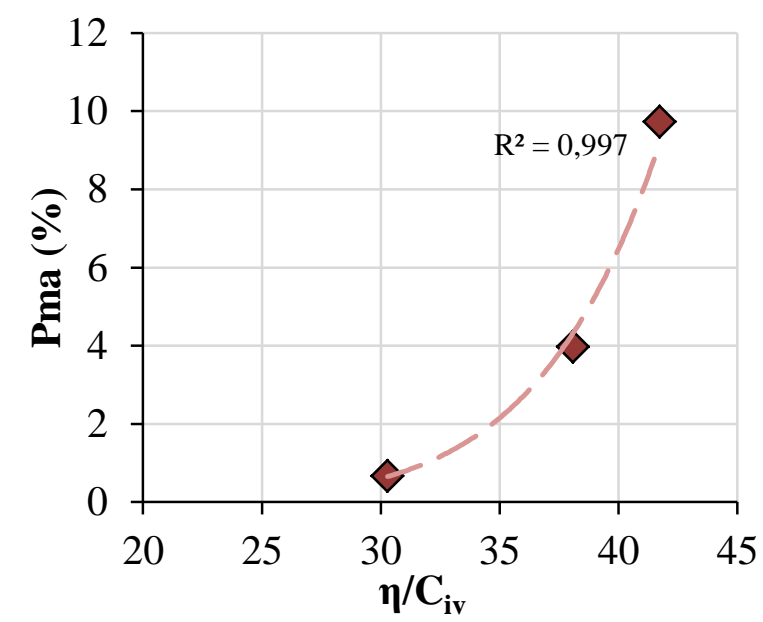

Fonte: $\mathrm{O}$ autor.

\section{CONCLUSÕES}

De acordo ao estabelecido na metodologia e nas análises dos resultados do presente trabalho pode-se concluir que existe uma influência direta da relação $\eta / C_{\text {iv }}$ na resistência à compressão simples das misturas solo-cimento estudadas, sendo possível criar uma equação de dosagem para estimar $q_{\mathrm{u}}$ (Equação 9) para qualquer tempo de cura, porosidade e teor de cimento desejados.

Adicionalmente, o aumento na resistência à compressão simples é diretamente proporcional ao teor de cimento usado, do tempo de cura e da porosidade das amostras, obtendo para o solo estudado valores máximos de $q_{4}=5000 \mathrm{kPa}$ usando $9 \% \mathrm{C}$ e $28-\mathrm{d}$ cura.

Foi comprovado que existe uma relação entre o índice porosidade/teor cimento e a perda de massa acumulada (Pma) das misturas. O uso do exponente 0,25 sobre 0 teor volumétrico de cimento permitiu estabelecer uma melhor tendência dos pontos experimentais das amostras submetidas a ensaios de compressão simples e assim correlacionar todos os tempos de cura através da relação $\eta / C_{i v}{ }^{0,25}$.
Finalmente, foi verificado de acordo às normas DNIT 143 (2010) e PCA (1992) que 5\% em massa é o teor mínimo de cimento recomendados para o uso do solo da camada cinza da FG para uso em pavimentação porque proporciona ganhos de resistência acima de 1200 kPa e Pma menores a 7-8\%.

\section{AGRADECIMENTOS}

O presente trabalho foi realizado com apoio da Coordenação de Aperfeiçoamento de Pessoal de Nível Superior - Brasil (CAPES) Código de Financiamento 001. O trabalho também foi realizado com o apoio do CNPq e da Fundação Araucária do Paraná.

\section{REFERÊNCIAS}

ASTM. ASTM D 2487 - 11 Standard Practice for Classification of Soils for Engineering Purposes (Unified Soil Classification System). 2011, ASTM International, West Conshohocken, Pa, D5521-5, 1-5.

ASTM. ASTM D 854 - 14 Standard Test Methods for Specific Gravity of Soil Solids by Water Pycnometer 1. 2014, ASTM International, West Conshohocken, Pa.

ASTM D559/D559M-15. Standard Test Methods for Wetting and Drying Compacted Soil-Cement Mixtures. ASTM International, 2015, West Conshohocken, Pa, 1-6.

ABNT - ASSOCIAÇÃO BRASILEIRA DE NORMAS TÉCNICAS Solos e rochas. NBR 6502: 1995. Rio de Janeiro, Brasil.

ABNT - ASSOCIAÇÃO BRASILEIRA DE NORMAS TÉCNICAS. Solo-Determinação do limite de liquidez. NBR 6459: 2016a. Rio de Janeiro, Brasil.

ABNT -ASSOCIAÇÃO BRASILEIRA DE NORMAS TÉCNICAS Solo - Determinação do limite de plasticidade. NBR 7180: 2016b, Rio de Janeiro, Brasil.

ABNT - ASSOCIAÇÃO BRASILEIRA DE NORMAS TÉCNICAS NBR 16605: Cimento Portland e 
outros materiais em pó -Determinação da massa específica. 2017, Rio de Janeiro, Brasil, 2017.

ABNT -ASSOCIAÇÃO BRASILEIRA DE NORMAS TÉCNICAS ABNT. NBR 7182: Solo - Ensaio de compactação. 2016c, Rio de Janeiro, Brasil.

ABNT - ASSOCIAÇÃO BRASILEIRA DE NORMAS TÉCNICAS ABNT. NBR 5739: Concreto - Ensaio de compressão de corpos de prova cilíndricos. 2018, Rio de Janeiro, Brasil.

BALDOVINO, J. A.; MOREIRA, E. B.; IZZO, R. L. DOS S.; ROSE, J. L. Empirical Relationships with Unconfined Compressive Strength and Split Tensile Strength for the Long Term of a Lime-Treated Silty Soil. Journal of Materials in Civil Engineering, v. 30, n. 8, p. 6018008, 2018a.

https://doi.org/10.1061/(ASCE)MT.1943$\underline{5533.0002378}$

BALDOVINO, J. A.; MOREIRA, E. B.; TEIXEIRA, W.; IZZO, R. L. S.; ROSE, J. L. Effects of lime addition on geotechnical properties of sedimentary soil in Curitiba, Brazil. Journal of Rock Mechanics and Geotechnical Engineering, 10(1), 188-194, 2018b. https://doi.org/10.1016/i.jrmge.2017.10.001

BALDOVINO, J. A.; MOREIRA, E. B.; IZZO, R. L. $S$. Discussion of Control factors for the long term compressive strength of lime treated sandy clay soil. Transportation Geotechnics, 15, 1-3, 2018c. https://doi.org/10.1016/i.trgeo.2017.11.002

BALDOVINO, J. D. J. A.; DOS SANTOS IZZO, R. L.; MOREIRA, E. B.; ROSE, J. L. Optimizing the evolution of strength for lime-stabilized rammed soil. Journal of rock mechanics and geotechnical engineering, v. 11 , n. 4, p. 882891 , 2019a. https://doi.org/10.1016/j.jrmge.2018.10.008

BALDOVINO, J. A.; MOREIRA, E. B.; CARAZZAI, É.; ROCHA, E.; IZZO, R.; MAZER, W.; ROSE, J. L. Equations controlling the strength of sedimentary silty soil-cement blends: influence of voids/cement ratio and types of cement. International Journal of Geotechnical Engineering, p. 1-14, 2019b. https://doi.org/10.1080/19386362.2019.161 $\underline{2134}$

BALDOVINO, J. D. J. A.; DOS SANTOS IZZO, R. L. Relação porosidade/cimento como parâmetro de control na estabilização de um solo siltoso. Colloquium Exactarum, v. 11, n. 1, p. 89-100, 2019. https://doi.org/10.5747/ce.2019.v11.n1.e269

BALDOVINO, J. D. J. A.; DOS SANTOS IZZO, R. L.; FELTRIM, F.; DA SILVA, É. R. Experimental Study on Guabirotuba's Soil Stabilization Using Extreme Molding Conditions. Geotechnical and Geological Engineering, 38, 2591-2607, 2020a. https://doi.org/10.1007/s10706-019-01171-x

BALDOVINO, J.; IZZO, R.; PEREIRA, M.; ROCHA, E.; ROSE, J.; BORDIGNON, V. Equations Controlling the Tensile and Compressive Strength Ratio of Sedimentary Soil-Cement Mixtures Under Optimal Compaction Conditions. Journal of Materials in Civil Engineering, v. 32, n. 1, p. 4019320, 2020b.

https://doi.org/10.1061/(ASCE)MT.1943-

$\underline{5533.0002973}$

CONSOLI, N. C.; FESTUGATO, L.; DA ROCHA, C. G.; CRUZ, R. C. Key parameters for strength control of rammed sand-cement mixtures: Influence of types of portland cement. Construction and Building Materials, v. 49, p. 591-597, 2013. https://doi.org/10.1016/i.conbuildmat.2013. $\underline{08.062}$

CONSOLI, N. C.; FOPPA, D.; FESTUGATO, L.; HEINECK, K. S. Key Parameters for Strength Control of Artificially Cemented Soils. Journal of Geotechnical and Geoenvironmental Engineering, v. 133, n. 2, p. 197-205, 2007. https://doi.org/10.1061/(ASCE)10900241(2007)133:2(197) 
DNIT - DEPARTAMENTO NACIONAL DE INFRAESTRUTURA DE TRANSPORTES . Pavimentação - Base de solo-cimento-. Especificação de serviço DNIT 143/2010. Rio de Janeiro, Brasil,2010.

DIAMBRA, A.; IBRAIM, E.; PECCIN, A.; CONSOLI, N. C.; AND FESTUGATO, L.. Theoretical Derivation of Artificially Cemented Granular Soil Strength. Journal of Geotechnical and Geoenvironmental Engineering, v. 143, n.5, p. 4017003, 2017. https://doi.org/10.1061/(ASCE)GT.19435606.0001646

DONRAK, J.; HORPIBULSUK, S.; ARULRAJAH, A.; KOU, H. L.; CHINKULKIJNIWAT, A.; HOY, $M$. Wetting-drying cycles durability of cement stabilised marginal lateritic soil/melamine debris blends for pavement applications. Road Materials and Pavement Design, v. 21, n.2, p. 500-518, 2020. https://doi.org/10.1080/14680629.2018.150 $\underline{6816}$

FIROOZI, A. A.; GUNEY OLGUN, C.; FIROOZI, A. A.; BAGHINI, M. S. Fundamentals of soil stabilization. International Journal of GeoEngineering, v. 8, n.1, p. 26, 2017. https://doi.org/10.1186/s40703-017-0064-9

MINEROPAR. Mapa Geológico do Paraná 1:250.000 e 1:650.000. Instituto de Terras, Cartografia e Geologia do Paraná - ITCG, $2020 . \quad$ Disponível em: http://www.mineropar.pr.gov.br/arquivos/Fil e/2_Geral/Geologia/PDF_Mapa_Geo_650000 /Mapa_Geologico_PR_650000_2006.pdf. Acesso em: 31 jan. 2020.

MOLA-ABASI, H.; SHOOSHPASHA, I. Influence of zeolite and cement additions on mechanical behavior of sandy soil. Journal of Rock Mechanics and Geotechnical Engineering, v. 8, p. 5, p. 746-752, 2016. https://doi.org/10.1016/i.jrmge.2016.01.008
IZZO, R. Effects of porosity, dry unit weight, cement content and void/cement ratio on unconfined compressive strength of roof tile waste-silty soil mixtures. Journal of Rock Mechanics and Geotechnical Engineering, v. 11 n. 2, p. 369-378, 2019. https://doi.org/10.1016/j.jrmge.2018.04.015

MOREIRA, E. B.; BALDOVINO, J. D. J. A.; DOS SANTOS IZZO, R. L.; ROSE, J. L. Impact of sustainable granular materials on the behavior sedimentary silt for road application. Geotechnical and Geological Engineering, v. 38, n. 1, p. 917-933, 2020. https://doi.org/10.1007/s10706-019-01025-6

PORTLAND CEMENT ASSOCIATION, PCA. Soilcement laboratory handbook. Skokie, IL: Portland Cement Association, 1992.

RIOS, S.; VIANA DA FONSECA, A.; BAUDET, B. A. Effect of the Porosity/Cement Ratio on the Compression of Cemented Soil. Journal of Geotechnical and Geoenvironmental Engineering, v.138, n.11, p. 1422-1426, 2012.

https://doi.org/10.1061/(ASCE)GT.19435606.0000698

MOREIRA, E. B.; BALDOVINO, J. A.; ROSE, J. L.; 